\title{
Neuer Bildungsplan Medizinische Praxisassistentln MPA EFZ
}

\section{Carlos Beat Quinto}

Dr. med., Departementsverantwortlicher Public Health und Gesundheitsberufe

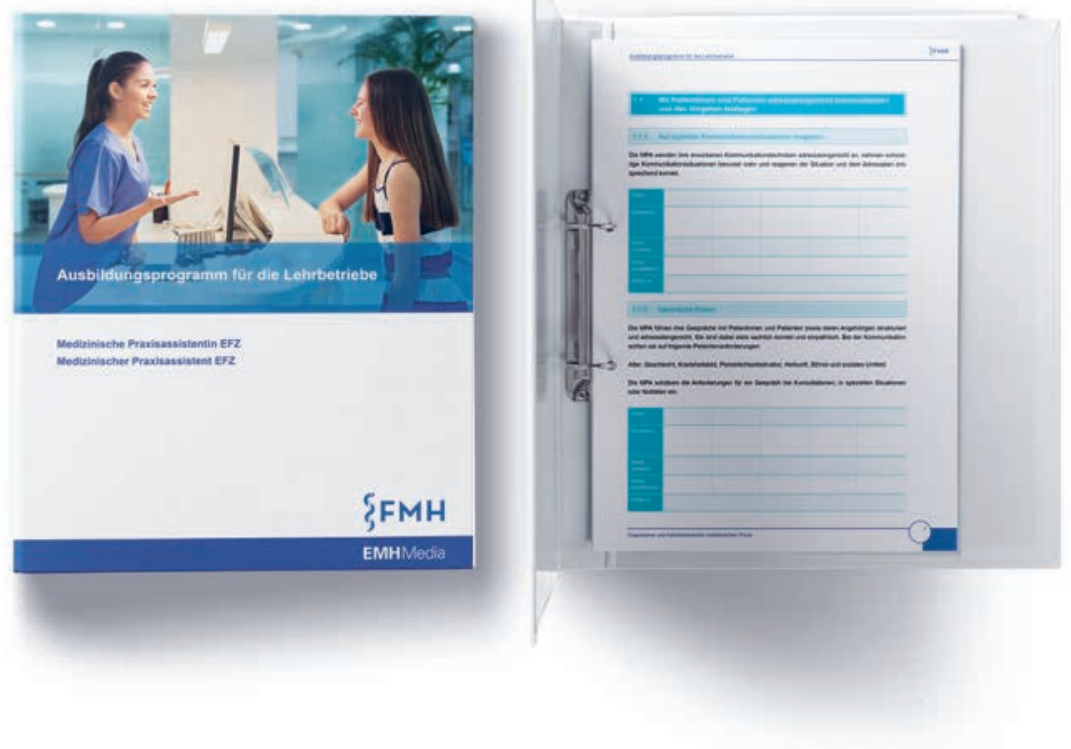

Ausbildungsprogramm für die Lehrbetriebe Medizinische Praxisassistentin EFZ

Medizinischer Praxisassistent EFZ

FMH Verbindung der Schweizer Ärztinnen und Ärzte (Hrsg.)

Basel: EMH Schweizerische Ärzteverlag AG; 2019.

Die Druckversion ist als Lose-Ordner-System verfügbar zum Preis von CHF 32.-/Stk.

Druckversion Deutsch: ISBN-Nr. 978-3-03754-126-5

Druckversion Französisch: ISBN-Nr. 978-3-03754-127-2

Druckversion Italienisch: ISBN-Nr. 978-3-03754-128-9

Die e-book-Version ist erhältlich zum Preis von CHF 20.-/Stk.

e-book-Version Deutsch: ISBN-Nr. 978-3-03754-129-6 e-book-Version Französisch: ISBN-Nr. 978-3-03754-130-2 e-book-Version Italienisch: ISBN-Nr. 978-3-03754-131-9

in eine abwechslungsreiche und anspruchsvolle berufliche Tätigkeit mündet.

Der Beruf der MPA ist sehr vielseitig und umfasst verschiedene Tätigkeitsfelder. Während der Ausbildung werden viele Kompetenzen erlernt, vertieft und gefestigt. Dieses Lehrmittel «Ausbildungsprogramm für die Lehrbetriebe» ermöglicht sowohl den Auszubildenden als auch den Ausbildnerinnen und Ausbildnern auf einfache Weise, sich zeit- und stufengerecht fortlaufend einen Überblick über die Kompetenzen der Auszubildenden zu verschaffen. Das Lehrmittel wurde somit auch als Arbeitsmittel geschaffen, gemäss aktuellen didaktischen Vorgaben. Pünktlich zum Beginn des neuen Schuljahres ist das «Ausbildungsprogramm für die Lehrbetriebe» als Druckversion und als e-book in allen drei Landessprachen verfügbar.

Ab Ausbildungsbeginn 2019 ist die MPA-Ausbildung Handlungskompetenzen-orientiert aufgebaut. So spricht man auch nicht mehr von Fächern, sondern von Handlungskompetenzen.
Die Bildungsverordnung (BiVo) und der Bildungsplan ische Praxisassistentin/Medizinischer wurden die BiVo und der Bildungsplan MPA EFZ in (SBFI) per 1. Januar 2019 in Kraft gesetzt.

Bildungsplan MPA EFZ beginnt im August 2019.

Aufgrund der Revision BiVo MPA EFZ wurde das Lehr mittel "Ausbildungsprogramm für die Lehrbetriebe" erarbeitet. Da sich die Medizin durch eine kurze wertszeit hinsichtlich neuen Wissens auszeichnet, waeine inhaltliche Erneuerung und Gewichtung der Ausbildungsinhalte erforderlich. Zudem können MPAEFZ-Lernende neu, bei Wunsch, berufsbegleitend eine Berufsmaturität ablegen. Dies war einerseits Vorgabe von Seiten des SBFI, andererseits dient dies auch dazu, die Attraktivität der Ausbildung MPA EFZ zu steigern. Bei entsprechend qualifizierten Lernenden soll so das Interesse für diese Ausbildung geweckt werden, welche 
Auf ein weiteres Lehrmittel, das ebenfalls neu erscheint, weisen wir hier gerne hin. Es handelt sich um den "Lehrplan für die Berufsfachschulen und Ausbildungsprogramm für die überbetrieblichen Kurse». Hauptzweck dieses Lehrplans ist die Vernetzung zwischen den Lernorten. Somit ist es wichtig, dass die Ausbildungsverantwortlichen in den Lehrbetrieben von diesem Lehrplan Kenntnis haben. Das theoretische Wissen und die praktischen Fertigkeiten werden den MPA-Lernenden während der Ausbildung an drei Orten vermittelt: in der Berufsfachschule (BFS), im Lehr- betrieb und in den überbetrieblichen Kursen, für deren Organisation, wahlweise in Zusammenarbeit mit weiteren Organisationen, die kantonalen Ärztegesellschaften verantwortlich sind. In einigen Kantonen/ Regionen wurden zwischenzeitlich «Lernortkoordinationskonferenzen" etabliert. Deren Besuch legen wir allen Ausbildungsverantwortlichen in den Lehrbetrieben sehr ans Herz. In der Regel werden diese Konferenzen durch die kantonalen Berufsbildungsämter organisiert.

\section{Lehrplan für die Berufsfachschulen und Ausbil-} dungsprogramm für die überbetrieblichen Kurse Medizinische Praxisassistentin EFZ

Medizinischer Praxisassistent EFZ

FMH Verbindung der Schweizer Ärztinnen und Ärzte (Hrsg.)

Basel: EMH Schweizerische Ärzteverlag AG; 2019.

Die Buchversion ist verfügbar zum Preis von CHF 49.-/Stk. Druckversion Deutsch: ISBN-Nr. 978-3-03754-124-1

Druckversion Französisch: ISBN-Nr. 978-3-03754-125-8

Die e-book-Version ist erhältlich zum Preis von CHF 35.-/Stk.

e-book-Version Deutsch: ISBN-Nr. 978-3-03754-132-6

e-book-Version Französisch: ISBN-Nr. 978-3-03754-133-3

Bestellungen des Ausbildungsprogramms für die Lehrbetriebe und des Lehrplans für die Berufsfachschulen und des Ausbildungsprogramms für die überbetrieblichen Kurse richten Sie bitte an: EMH Schweizerischer Ärzteverlag, Basel: auslieferung[at]emh.ch

Weitere Informationen zur Ausbildung MPA EFZ und Informationen für Arbeitgeber finden Sie auf der Homepage www.mpaschweiz.ch 